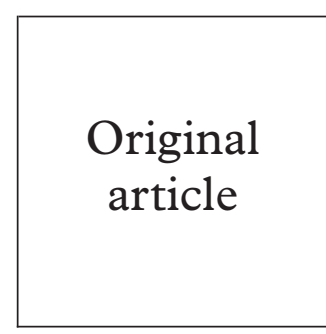

\title{
Chlamydia trachomatis prevalence and sexual behaviour among female adolescents in Belgium
}

\author{
B Vuylsteke, M Vandenbruaene, P Vandenbulcke, E Van Dyck, M Laga
}

Objectives: To determine prevalence and risk factors of Chlamydia trachomatis among female secondary school students and to develop potential selective screening strategies.

Methods: A cross sectional survey was conducted in the 17 school medical centres in Antwerp municipality, Belgium. All female students of forms 5 or 6 , who were due for their medical check up during the school year 1996-7, were invited to participate. A self administered questionnaire on general and sexual behaviour, and a first void urine sample were collected. The urine specimen was tested for $C$ trachomatis with ligase chain reaction assay, and positive tests were confirmed with polymerase chain reaction assays.

Results: 2784 female students participated in the study. Their median age was 17 , and $52 \%$ of them reported having sexual intercourse at least once. The prevalence of $C$ trachomatis among sexually active women was $1.4 \%$. Factors significantly associated with infection in multivariate analysis were number of lifetime partners, genital complaints of partner, type of secondary school, and a history of pregnancy. Selective screening of those women who are at highest risk for infection would have detected $90 \%$ of all infections, and require testing of $14 \%$ to $18 \%$ of the population.

Conclusions: The prevalence of $C$ trachomatis was relatively low among female secondary school students in Antwerp, but unsafe sex practices were evident because of the high number of unplanned pregnancies. Selective screening strategies with a high sensitivity can be proposed, but should be assessed for acceptability, feasibility, and cost.

(Sex Transm Inf 1999;75:152-155)

Keywords: sexual behaviour; female adolescents; screening strategies; Chlamydia trachomatis; Belgium

\section{Introduction}

Chlamydia trachomatis is the most common bacterial sexually transmitted infection in Europe. ${ }^{1}$ Clinical signs and symptoms of genital chlamydial infection can be mild or non-specific, and as many as $70 \%$ of infected women may remain without symptoms. ${ }^{2}$ Complications resulting from untreated chlamydial infection include pelvic inflammatory disease (PID), infertility, and ectopic pregnancy. ${ }^{2}$ These costly and potentially serious lifetime consequences call for the control of chlamydial infection as an important public health measure.

The prevalence of $C$ trachomatis in the general population has not been accurately determined, largely because of the asymptomatic nature of infection and the need for endocervical swabs to establish the diagnosis. Recently, DNA amplification tests such as ligase chain reaction (LCR) or polymerase chain reaction (PCR) have become available and show high sensitivity and specificity when used on urine samples, enabling widespread screening for $C$ trachomatis infection. ${ }^{34}$

In Belgium, there is currently no generalised screening policy for $C$ trachomatis infection, and prevalence data from the general population are not available. Since young women are at particularly high risk for $C$ trachomatis infection and are a priority target group for prevention and control activities, we decided to set up a survey among 16-18 year old young women. All secondary school students in Belgium have to have a routine medical check up every 2 years. This check up takes place in specialised school medical centres, which is where this study was undertaken.

The objectives of the present study were to determine the prevalence and risk factors for chlamydial infection among female secondary school students in Antwerp, and to design potential screening strategies based on risk factors for infection.

\section{Methods}

STUDY DESIGN

The study was conducted during the school year 1996-7 in the 17 school medical centres of Antwerp municipality. All female students of forms 5 or 6 (16-18 years old) who were due for their medical check up were invited to participate. Information on the study was initially given through a letter, which was sent to both the students and their parents. A video was shown in the waiting room of the medical centre, providing general information on $C$ trachomatis infection and specific information on the study procedures. All the female students who wished to participate were then given some privacy in order to fill in an anonymous questionnaire which included questions on sexual behaviour and genital symptoms. A first void urine sample was then collected by each participant in a urine container, labelled with the same unique study number as the questionnaire number. An identification card containing the study number was taken home by each 
Table 1 General and sexual behaviour characteristics of female secondary school students in Antwerp, Belgium ( $n=2784)$

\begin{tabular}{|c|c|c|c|c|}
\hline & $\begin{array}{l}\text { All } \\
(n=2784) \%\end{array}$ & $\begin{array}{l}\text { Sexually active } \\
(n=1433) \%\end{array}$ & $\begin{array}{l}\text { Non-sexually active } \\
(n=1307) \%\end{array}$ & $p$ Value ${ }^{\star}$ \\
\hline \multicolumn{5}{|l|}{ Age } \\
\hline $15-16$ & 16.5 & 11.9 & 21.3 & \\
\hline 17 & 48.2 & 41.6 & 55.8 & \\
\hline 18 & 23.8 & 29.9 & 16.9 & \\
\hline $19-23$ & 11.5 & 16.6 & 6.0 & $<0.001$ \\
\hline Belgian nationality & 87.3 & 91.5 & 83.5 & $<0.001$ \\
\hline \multicolumn{5}{|l|}{ Type of secondary school } \\
\hline General & 40.3 & 30.4 & 51.8 & \\
\hline Art & 5.8 & 6.4 & 5.3 & \\
\hline Technical & 30.4 & 37.2 & 23.2 & \\
\hline Vocational & 23.5 & 26.0 & 19.7 & 0.001 \\
\hline Currently smoking & 38.5 & 55.2 & 20.6 & $<0.001$ \\
\hline Drinking alcohol regularly† & 35.1 & 42.1 & 27.3 & $<0.001$ \\
\hline History of genital symptoms $\ddagger$ & 48.5 & 59.9 & 36.3 & $<0.001$ \\
\hline Current genital symptoms & 17.9 & 22.5 & 13.0 & $<0.001$ \\
\hline Current antibiotic use & 6.0 & 6.0 & 6.0 & 0.94 \\
\hline Prevalence Chlamydia trachomatis & 0.8 & 1.4 & 0.0 & $<0.001$ \\
\hline \multicolumn{5}{|l|}{ Age at first sexual intercourse $\$$} \\
\hline$<15$ & & 14.7 & & \\
\hline 15 & & 23.7 & & \\
\hline 16 & & 33.7 & & \\
\hline$\geqslant 17$ & & 27.8 & & \\
\hline \multicolumn{5}{|c|}{ Number of sexual partners during past 12 months $\rrbracket$} \\
\hline 1 & & 75.2 & & \\
\hline 2 & & 16.7 & & \\
\hline$\geqslant 3$ & & 8.0 & & \\
\hline \multicolumn{5}{|l|}{ Number of lifetime sexual partners $\$$} \\
\hline 1 & & 45.7 & & \\
\hline 2 & & 22.2 & & \\
\hline 3 & & 13.1 & & \\
\hline$\geqslant 4$ & & 14.5 & & \\
\hline Don't remember & & 4.5 & & \\
\hline \multicolumn{5}{|l|}{ Condom use $₫$} \\
\hline Consistent (always) & & 20.6 & & \\
\hline During first sexual contact & & 80.0 & & \\
\hline During last sexual contact & & 36.5 & & \\
\hline \multicolumn{5}{|l|}{ Current contraceptive use $₫$} \\
\hline None & & 9.6 & & \\
\hline Pill only & & 51.6 & & \\
\hline Condoms only & & 21.6 & & \\
\hline Pill + condoms & & 17.1 & & \\
\hline History of pregnancy $\$$ & & 3.5 & & \\
\hline
\end{tabular}

${ }^{\star} \chi^{2}$ test for differences in proportion between sexually active and non-sexually active adolescents. †Regularly was defined as drinking alcohol during the weekend or several days a week.

‡Genital symptoms were defined as vaginal itching, vaginal discharge, or intermenstrual bleeding. Questions only asked to those who reported to be sexually active.

participant, allowing her to obtain her results anonymously by phoning the Institute of Tropical Medicine or the school medical centre, after 3 weeks. If the results were positive, participants were instructed to consult their general practitioner, their gynaecologist, or to visit the sexually transmitted infections clinic of the Institute of Tropical Medicine. All general practitioners and gynaecologists in the Antwerp region had been informed about the study by letter. They had been advised to treat women with a positive result, without retesting them, with a single dose of azithromycin or a 10 day regimen of doxycycline, and to treat the sexual partners as well. Participants with a confirmed $C$ trachomatis infection who failed to phone for their result within 6 weeks after the examination day were traced by birth date and collection date and were confidentially informed of the result by the medical personnel of the school medical centre. The study has received approval from the ethics committee of the Institute of Tropical Medicine.

LABORATORY TESTS

The urine samples were refrigerated at $2-8^{\circ} \mathrm{C}$ during storage and transported to the laboratory of microbiology at the Institute of Tropical Medicine within 24 hours. Urine samples were tested with the plasmid based LCR assay
Table 2 Risk factors associated with Chlamydia trachomatis (CT) in sexually active female adolescents in Antwerp, Belgium

\begin{tabular}{|c|c|c|c|}
\hline Characteristics & $N$ & $\% C T$ & $p$ Value \\
\hline \multicolumn{4}{|l|}{ Age } \\
\hline $15-16$ & 163 & 0.0 & \\
\hline 17 & 575 & 1.6 & \\
\hline 18 & 410 & 1.5 & \\
\hline $19-23$ & 228 & 2.2 & $0.01^{\star}$ \\
\hline \multicolumn{4}{|c|}{ Type of secondary school } \\
\hline General & 420 & 0.0 & \\
\hline Art & 87 & 1.1 & \\
\hline Technical & 510 & 2.4 & \\
\hline Vocational & 363 & 1.9 & 0.02 \\
\hline \multicolumn{4}{|l|}{ Nationality of mother } \\
\hline Belgian & 1196 & 1.1 & \\
\hline Not Belgian & 181 & 3.9 & 0.01 \\
\hline \multicolumn{4}{|c|}{ History of vaginal discharge } \\
\hline Yes & 351 & 2.6 & \\
\hline No & 921 & 1.0 & 0.04 \\
\hline \multicolumn{4}{|c|}{ Current symptom of vaginal discharge } \\
\hline Yes & 188 & 3.2 & \\
\hline No & 1108 & 1.1 & 0.04 \\
\hline \multicolumn{4}{|c|}{ History of intermenstrual bleeding } \\
\hline Yes & 221 & 3.6 & \\
\hline No & 1024 & 1.0 & 0.007 \\
\hline \multicolumn{4}{|c|}{ Partner had a genital complaint in past 3 months $\dagger$} \\
\hline Yes & 41 & 9.8 & \\
\hline No & 872 & 1.1 & 0.002 \\
\hline \multicolumn{4}{|c|}{ Age of first sexual intercourse } \\
\hline$<16$ years & 514 & 2.3 & \\
\hline$\geqslant 16$ years & 856 & 0.9 & 0.04 \\
\hline \multicolumn{4}{|c|}{ Number of sexual partners in the past 12 months } \\
\hline 1 & 979 & 0.7 & \\
\hline$\geqslant 2$ & 320 & 4.1 & $<0.001$ \\
\hline \multicolumn{4}{|c|}{ Number of lifetime partners } \\
\hline $1-2$ & 924 & 0.2 & \\
\hline$>2$ & 373 & 3.7 & \\
\hline Don't remember & 60 & 6.7 & $<0.001$ \\
\hline \multicolumn{4}{|l|}{ Condom use } \\
\hline Always & 284 & 0.0 & \\
\hline Regularly & 197 & 1.5 & \\
\hline Sometimes & 373 & 2.9 & 0.02 \\
\hline Never & 505 & 1.2 & $0.18^{\star}$ \\
\hline \multicolumn{4}{|l|}{ History of pregnancy } \\
\hline Yes & 49 & 8.2 & \\
\hline No & 1307 & 1.1 & 0.003 \\
\hline
\end{tabular}

${ }^{\star} \chi^{2}$ for trend.

†Genital complaint in partner was defines as dysuria, urethral discharge, or genital warts.

(LCX, Abbott Laboratories, Chicago, IL, USA) according to the manufacturer's instructions. Samples which tested positive with the LCR assay were retested with the PCR assay (Amplicor, Roche, Diagnostic Systems, Branchburg, USA). Only samples which tested positive on both LCR and PCR assay were considered positive. Internal validation was done by retesting 200 samples of high risk participants with an initially LCR negative result.

STATISTICAL METHODS

Statistical analysis was performed using Intercooled STATA 5.0 statistical package. Univariate analysis was done using Yates's corrected $\chi^{2}$ or Fisher's exact test for comparing proportions and Student's $t$ test or Kruskal-Wallis test for comparing means and medians. Independent risk factors of chlamydial infections were assessed using stepwise logistic regression, with variables entered into multivariate analysis on the basis of significance $(p<0.05)$ in univariate analysis.

VALIDATION OF SCREENING STRATEGIES

After finishing the main study and the risk factor analysis, the results were used to validate different screening strategies. These strategies were designed based on risk factors for 
Table 3 Logistic regression analysis of factors associated with chlamydial infection

\begin{tabular}{lll}
\hline & OR $(95 \%$ CI $)$ & $p$ Value \\
\hline Type of school & $2.3(1.02-5.01)$ & 0.04 \\
Current symptom of vaginal discharge & $3.0(0.72-12.4)$ & 0.13 \\
Partner had a genital complaint in past 3 months & $14.2(3.17-63.90)$ & 0.001 \\
Sexual debut <16 years & $4.3(0.98-19.07)$ & 0.05 \\
Number of sex partners in past 12 months & $3.4(0.73-15.86)$ & 0.12 \\
Number of lifetime sex partners & $6.6(1.86-23.50)$ & 0.004 \\
History of pregnancy & $6.7(1.08-41.49)$ & 0.04 \\
\hline
\end{tabular}

Number of cases with complete data $=729$.

chlamydial infection, which were feasible in a school health setting. A first method consisted of combining risk factors obtained in the univariate analysis, which had a sensitivity of $70 \%$ or more for detecting infection. Another method involved building up a personal risk score for each of the sexually active participants. Independent risk factors from the multivariate analyses were selected. Points were assigned to each risk factor, with a value of the adjusted odds ratio divided by four and rounded to the nearest whole number. The score for each woman was calculated as the sum of the points for each risk factor present. Different cut off values were chosen, resulting in a decision to test. The outcomes of the strategies developed by both approaches were then simulated using data from the questionnaire and the results of the laboratory tests. The following indicators were calculated: prevalence of $C$ trachomatis in the selected group (positive predictive value), and the proportion of infections detected by the screening strategy (sensitivity of the strategy for detecting infection). We aimed to achieve a screening strategy with a combination of a high sensitivity (at least $80 \%$ ) and an acceptable positive predictive value, which was defined as $3 \%$ or higher, according to the results of cost effectiveness studies in the United States. ${ }^{5}$

\section{Results}

CHARACTERISTICS OF THE STUDY POPULATION Overall, 3169 female students in forms 5 or 6 of secondary education in Antwerp municipality were due for their medical check up in the

Table 4 Results of different selective screening strategies for Chlamydia trachomatis (CT) infection

\begin{tabular}{llll}
\hline $\begin{array}{l}\text { Combination of risk criteria } \\
\text { \% of the population to }\end{array}$ & CT prevalence (\%) & $\begin{array}{l}\text { Sensitivity of the } \\
\text { strategy (\%) }\end{array}$ \\
\hline $\begin{array}{l}\text { Test everybody } \\
\text { Test if: }\end{array}$ & 100 & 0.7 & 100 \\
$\quad$ (a) & 52.6 & 1.4 & 100 \\
(a) + (b) & 41.0 & 1.9 & 100 \\
(a) + (b) + (c) & 26.3 & 2.1 & 75 \\
(a) + (b) + (d) & 16.9 & 2.7 & 60 \\
(a) + (b) + (e) & 10.3 & 4.7 & 65 \\
(a) + (b) + (f) & 14.5 & 4.6 & 90 \\
Personal risk score* in sexually active women & & \\
Test is risk score: & & \\
$\geqslant 1$ & 26.7 & 2.5 & 90.0 \\
$\geqslant 2$ & 18.1 & 3.7 & 90.0 \\
$\geqslant 3$ & 11.7 & 4.2 & 65.0 \\
\hline
\end{tabular}

(a) Sexually active.

(b) Not consistent condom use.

(c) History of vaginal discharge or intermenstrual bleeding or vaginal itching.

(d) Sexual debut $<16$ years.

(e) $\geqslant 2$ sexual partners in the past 12 months.

(f) $>2$ lifetime sexual partners or don't remember the number of lifetime partners.

*Personal risk is the sum of following points for each woman: if partner symptomatic, 3 points; if more than two lifetime sexual partners or don't remember number, 2 points; if history of pregnancy, 2 points; if sexual debut $<16$ years, 1 point. school year 1996-7 (Flemish Ministry of Education). Of these, $2784(88 \%)$ agreed to participate in the study. Sexually active women represented $52 \%$ of the total study population. Demographic and reproductive health characteristics of sexually active and non-sexually active participants are summarised in table 1 . There was a difference between sexually active participants and participants who were not sexually active as regards age, nationality, and type of education. Of all women who reported past or current symptoms, $38 \%$ had consulted a physician for these complaints.

The prevalence of $C$ trachomatis was $1.4 \%$ (20/1380) among sexually active women and $0 \%(0 / 1244)$ among women who had never been sexually active. Sexual behaviour characteristics of sexually active participants are also presented in table 1 . The median age at first sexual intercourse was 16 years, and more than half of the women reported more than one lifetime partner. Condoms were used during the first sexual contact by $80 \%$ of the participants, although only $21 \%$ used a condom consistently. Ninety per cent of all sexually active participants reported current use of any contraceptive method and $3.5 \%$ reported a history of pregnancy.

RISK FACTORS FOR C TRACHOMATIS INFECTION Table 2 summarises characteristics which were significantly associated with chlamydial infection in univariate analysis, including age (trend with increasing age), type of school (students from general school less infected), non-Belgian nationality of the mother, history of vaginal discharge, current symptom of vaginal discharge, history of intermenstrual bleeding, genital complaints of sexual partner, age at first sexual intercourse less than 16 years, having more than one sexual partner in the past 12 months, more than two lifetime sexual partners, inconsistent condom use, and a history of pregnancy. In multivariate analysis (table 3), the following factors remained significantly associated with infection: type of school, genital complaints of partner, number of lifetime partners, and a history of pregnancy. The nationality of the mother was not associated with infection in multivariate analysis.

OPTIONS FOR SELECTIVE SCREENING STRATEGIES Different selective screening strategies were defined based on risk factor analysis and were validated in a desk study (see methods). When using one or a combination of risk factors as criteria for testing, no strategy could be detected which combined a sensitivity of $100 \%$ and a prevalence of $C$ trachomatis of more than $3 \%$ (table 4 ). The optimal approach in terms of a high sensitivity combined with a low number of women to be tested was to test all female adolescents who were sexually active, did not use condoms consistently, and who had more than two lifetime partners or did not remember the number (strategy (a) + (b) + (f)). Using this strategy, only $14 \%$ of the population would have to be tested and $90 \%$ of all infections would be detected. Another approach consisted of building a personal risk score for each 
of the sexually active participants, based on the following factors: if partner had genital complaints, 3 points; if more than two lifetime sexual partners or did not remember the number, 2 points; if history of pregnancy, 2 points; if sexual debut $<16$ years, 1 point. When selecting women with a risk score of 2 and more for testing, $90 \%$ of all infections would be detected, and $18 \%$ of the total population would have to be tested.

\section{Discussion}

The prevalence of $C$ trachomatis among female, sexually active, secondary school students in Antwerp was $1.4 \%$. The study population can be considered representative for all secondary school students in forms 5 or 6 in the Antwerp region, as school medical examinations are compulsory in Belgium. This prevalence $(1.4 \%)$ may seem low, especially when comparing with prevalences found in recent European studies. In London, for instance, the prevalence of $C$ trachomatis among asymptomatic women aged 18-35 years attending general practices was $2.5 \% .{ }^{6}$ In Amsterdam, women were invited to send in a urine sample by mail, and $13.4 \%$ of the women aged 16-20 years were infected with $C$ trachomatis.

The type of school was associated with infection, and was still associated significantly when adjusting for the number of sexual partners. Students attending general secondary school, as opposed to technical and nonacademic schools, even those with a high risk profile (more than two sexual partners) were not infected. Early studies in HIV infection focused on numbers of sexual partners as a key variable, but there is a growing debate about the importance of different network variables. Mixing patterns between different residential or occupational groups may explain how large differences in HIV seropositivity between neighbouring localities can be maintained for long periods. ${ }^{89}$ This theory could also be applied to explain differences in $C$ trachomatis prevalence among students from different types of schools in the same geographic area. Assuming that students from one type of school remain in the same network for sport and other social events, it is highly possible that they stay in that network for sexual contacts as well. These distinct sexual networks would contribute to the absence of infection in highly sexually active students in general secondary school.

Unsafe sex puts a woman at risk of both sexually transmitted infections and unwanted pregnancy. In our study, the number of adolescents reporting a history of pregnancy was more than twice the number of adolescents infected with $C$ trachomatis. These results emphasise the particular vulnerability of this group to unplanned pregnancy and make a strong case for enhancing sexual and reproductive health education, both in the health education curriculum of the secondary schools and in the medical centres.

Universal screening is no longer cost effective if prevalence is under 3\% (United States) or under $6 \%$ (Sweden). ${ }^{5}$ Based on the study results, screening criteria were designed in order to identify a subgroup of students, in which the majority of infections would be detected. The cost effectiveness of these strategies should be analysed based on the real costs in the Belgian situation. Also, the acceptability and feasibility of different selective screening strategies should be assessed in a pilot study before starting a widespread $C$ trachomatis screening programme in secondary schools in Belgium.

In conclusion, the prevalence of $C$ trachomatis was relatively low among female secondary school students in Antwerp, but unsafe sex practices were demonstrated by the high number of unplanned pregnancies, calling for strengthening of sexual health education in schools. Selective screening strategies with a high sensitivity can be proposed, but should be assessed for acceptability, feasibility, and cost.

This study was funded by the Flemish Fund for Scientific Research and the Flemish Ministry of Public Health

The authors would like to thank the personnel of all school medical centres in Antwerp for their continuous support during the study, Central Laboratory of Antwerp for transporting the the study, Central Laboratory of Antwerp for transportin
urine samples, and Pfizer for providing free treatment.

Contributions: Bea Vuylsteke, principal investigator; Marc Contributions: Bea Vuylsteke, principal investigator; Marc
Vandenbruaene, field coordinator; Pieter Vandenbulcke, coordinator for the school medical centres of Antwerp municipality; Eddy Van Dyck, laboratory supervisor; Marie Laga, study team supervisor.

1 Piot P, Islam M. Sexually transmitted diseases in the 1990 s. Sex Transm Dis 1994;21:S7-13.

2 Cates W, Wasserheit JN. Genital chlamydial infections: epidemiology and reproductive sequelae. Am f Obstet Gynecol demiology and rep

3 Lee HH, Chernesky MA, Schachter J, et al. Diagnosis of Chlamydia trachomatis genitourinary infection in women by ligase chain reaction assay of urine. Lancet 1995;345: 213-16.

4 Schachter J, Moncada J, Whidden R, et al. Noninvasive tests for diagnosis of Chlamydia trachomatis infection: application of ligase chain reaction to first-catch urine specimens of women. $\mathcal{F}$ Infect Dis 1995;172:1411-14

5 Marrazzo JM, Celum CL, Hillis SD, et al. Performance and cost-effectiveness of selective screening criteria for cor 1997;24:131-41.

6 Grun L, Tassano-Smith J, Carder C, et al. Comparison of two methods of screening for genital chlamydial infection in women attending general practice: cross sectional survey.

7 van den hoek JA, Mulder Folkerts D, Coutinho RA, et al. Opportunistic screeningsprogram for CT infection in Amsterdam, the Netherlands. International Congress of STD, Seville, Spain, 1997:O257.

8 Barlow D, Daker-White G, Band B. Assortive sexual mixing in a heterosexual clinic population - a limiting factor in HIV spread? AIDS 1997;11:1039-44.

9 Pickering $\mathrm{H}$, Okongo $\mathrm{M}$, Ojwiya $\mathrm{A}$, et al. Sexual networks in Uganda: mixing patterns between a trading town, its rural hinterland and a nearby fishing village. Int $¥$ STD AIDS 1997;8:495-500. 